\title{
Logistic regression analysis and a risk prediction model of pneumothorax after CT-guided needle biopsy
}

\author{
Yanfeng Zhao ${ }^{1 *}$, Xiaoyi Wang ${ }^{1 *}$, Yong Wang ${ }^{2}$, Zheng Zhu ${ }^{1}$ \\ ${ }^{1}$ Department of Diagnostic Radiology, ${ }^{2}$ Department of Ultrasound, National Cancer Center/Cancer Hospital, Chinese Academy of Medical Sciences \\ and Peking Union Medical College, Beijing 100021, China \\ Contributions: (I) Conception and design: Y Zhao, Y Wang; (II) Administrative support: Y Wang; (III) Provision of study materials or patients: Y \\ Zhao; (IV) Collection and assembly of data: X Wang, Z Zhu; (V) Data analysis and interpretation: X Wang, Z Zhu; (VI) Manuscript writing: All \\ authors; (VII) Final approval of manuscript: All authors. \\ *These authors contributed equally to this work. \\ Correspondence to: Yong Wang. Department of Ultrasound, National Cancer Center/Cancer Hospital, Chinese Academy of Medical Sciences and \\ Peking Union Medical College, Beijing 100021, China. Email: drwangyong77@163.com.
}

Background: Pneumothorax is the most common complication of computed tomography (CT)-guided needle biopsy. The purpose of this study was to investigate independent risk factors of pneumothorax, other than emphysema, after CT-guided needle biopsy and to establish a risk prediction model.

Methods: A total of 864 cases of CT-guided needle biopsy with an 18-gauge cutting needle were enrolled in this study. The relevant risk factors associated with pneumothorax included age, sex, emphysema, shortaxis size of the lesion, depth of the lesion, body position, and the number of pleural punctures. Several independent risk factors of pneumothorax were found, and a predictive model for pneumothorax was established using univariate and multivariate logistic regression analyses.

Results: Pneumothorax occurred in 31.4\% (271/864) of cases. Univariate analysis showed that significant risk factors of pneumothorax included age, emphysema, small lesion size, no contact between the lesion and the pleura, prone or lateral body position, and multiple punctures. Independent risk factors of pneumothorax in the multivariate logistic regression analysis included emphysema $(\mathrm{P}=0.000)$, no contact between the lesion and the pleura $(\mathrm{P}=0.000)$, prone or lateral body position $(\mathrm{P}=0.002)$, and the number of pleural punctures $(\mathrm{P}=0.000)$. The sensitivity, specificity, and accuracy of the predictive model for pneumothorax were $56.8 \%$, $79.6 \%$, and $72.5 \%$, respectively.

Conclusions: Pneumothorax is a common complication of CT-guided lung biopsy. Independent risk factors of pneumothorax include emphysema, no contact between the lesion and the pleura, and prone or lateral body position. The predictive model developed in this study was highly accurate in predicting the incidence of pneumothorax.

Keywords: Computed tomography; CT-guided needle biopsy; multivariate logistic regression analysis; pneumothorax; risk factor

Submitted Aug 05, 2017. Accepted for publication Sep 07, 2017.

doi: $10.21037 /$ jtd.2017.09.47

View this article at: http://dx.doi.org/10.21037/jtd.2017.09.47

\section{Introduction}

Computed tomography (CT)-guided needle biopsy was first reported by Haaga in 1976 (1), and with advances in technology, CT has become the primary guidance technique for transthoracic needle biopsies, especially for lesions that other imaging devices cannot visualize (2-11). Potential complications of CT-guided needle biopsy include pneumothorax, hemoptysis, infection, implant transfer, and air embolism (12-20). Pneumothorax is a common and 
potentially life-threatening complication of puncturing the thorax. Emphysema has been widely recognized as an independent risk factor of pneumothorax after biopsy; however, other risk factors, such as lesion size, lesion depth, and patient age remain controversial (15,16,21-26). Although most researchers believe that the size and depth of the lesion as well as patient age are risk factors, there is no published evidence definitively supporting this. Thus, the purpose of our study was to investigate independent risk factors, other than emphysema, of pneumothorax after CT-guided needle biopsy and to establish a risk prediction model.

\section{Methods}

\section{Patients}

This prospective study was approved by the Ethics Committee of our hospital, and all 864 patients who underwent a CT-guided needle biopsy from October 2011 to June 2016 signed an informed consent before the procedure. All patients underwent a bronchoscopic biopsy with negative results prior to the CT-guided procedure. All patients also underwent a chest CT scan within 28 days before the biopsy, and an enhanced CT scan was performed if the lesion was close to vascular structures on the initial plain CT scan. Blood tests, including evaluation of platelet count, prothrombin time, and partial thromboplastin time, were performed in all patients within 7 days before the biopsy. If the patient was taking aspirin, the biopsy was performed at least 7 days after discontinuing the medication.

\section{Materials}

All patients underwent a 64-slice spiral CT (Toshiba Aquilion, Nasu, Japan) with a thickness of $5 \mathrm{~mm}$, a layer spacing of $5 \mathrm{~mm}$, a tube voltage of $120 \mathrm{kV}$, and a tube current of $50 \mathrm{~mA}$. The skin puncture point and needle path were chosen based on the shortest distance between the puncture point and the lesion that avoided bone, bullae, and vessels. The choice of body position, such as supine, prone, or lateral, was selected according to the needle path and skin puncture point. A disposable puncture needle with a semi-automatic ejection biopsy device (NIPRO ELPICK, Plastic Honda Co., Ltd., Tokyo, Japan) was used. The size of the needle was 18 -gauge; a needle length of 10,15 , or $20 \mathrm{~cm}$ was selected according to the depth of the biopsy; a coaxial needle was not used in any of the biopsies.

\section{Biopsy procedure}

A CT scan was performed with patients holding their breath, and the skin surface projection area of the lesion was located. Scanning the puncture area again after placing a marker on the skin surface, the needle path was determined, including depth, angle, and skin puncture point. The skin near the puncture area was disinfected with iodophor, a sterile operating drape was placed, and local anesthesia was injected from the skin to the pleura with $5 \mathrm{~mL}$ of $2 \%$ lidocaine hydrochloride. A CT scan was again preformed in this area when the needle passed through the pleura, and the needle was inserted into the lesion with the patient holding his or her breath. The patient was scanned again to ensure that the needle tip was within the lesion. The needle core was then removed and exchanged for an ejection biopsy device. Tissue specimens were extracted and placed into a $10 \%$ formaldehyde solution for pathological examination. Multiple biopsies were performed depending upon the tissue specimen and purpose of the biopsy. All biopsies were performed by a single radiologist with over five years of CT-guided needle biopsy experience.

A CT scan was performed immediately after the biopsy followed by a chest radiographic examination $2 \mathrm{~h}$ later to determine if a pneumothorax was present. In the event of a pneumothorax, chest radiographs were acquired again in $2 \mathrm{~h}$ if the pneumothorax was small, or a chest tube was placed immediately if the pneumothorax was large (Figure 1).

\section{Statistical analysis}

Eight risk factors of pneumothorax were used for the univariate and multivariate analysis based on the literature (16,26-30) and clinical experience as follows: (I) patient: age, sex, and history of emphysema; (II) lesion: size [shortaxis diameter: measured with a lung window width of 1,600 Hounsfield units (HU) and a window level of $-600 \mathrm{HU}$ ], depth (the depth of normal lung tissue along the needle path and contact between the lesion and the pleura); (III) biopsy: body position (supine, prone, or lateral) and number of pleural punctures.

The chi-square or Fisher's exact test was used for single factor analysis of categorical variables. Single factor analysis of continuous variables was performed with the $t$-test, receiver operating characteristic curve (ROC curve), and the Youden index (YI); measurements corresponding to the maximum YI value were considered the best diagnostic values. All risk factors were analyzed via multivariate logistic 


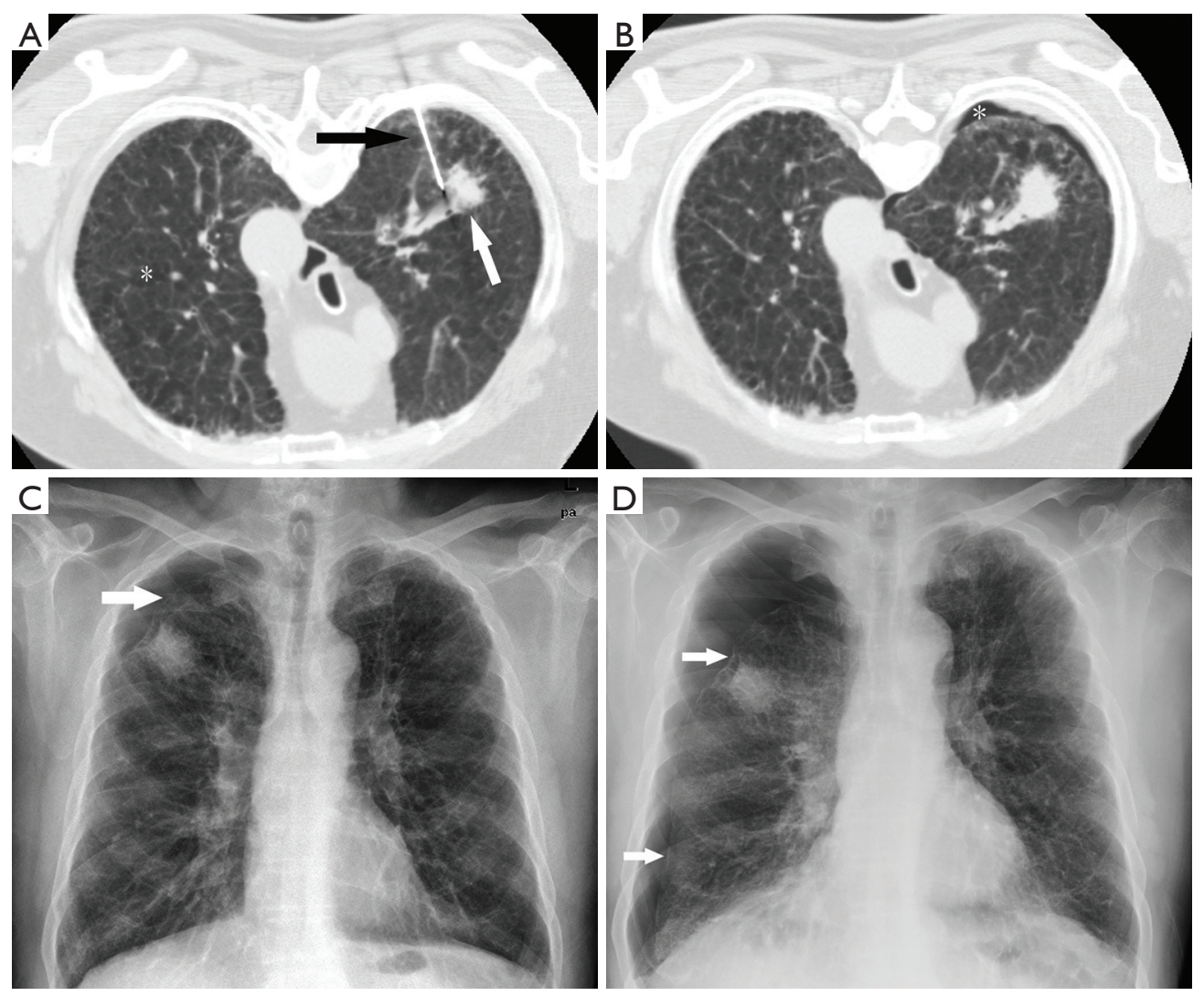

Figure 1 Male, 72-year-old, with needle biopsy of the nodule on the right upper lobe. (A) Bilateral emphysema $\left(^{*}\right)$ was showed on the axial prone position CT image and the needle (black arrow) was through the pulmonary tissue, white arrow indicates lesion; (B) the pneumothorax in right chest $\left(^{*}\right)$ was showed on CT scan after biopsy; (C) the pneumothorax (white arrow) was showed on the chest radiograph after 1 h of biopsy; (D) after $6 \mathrm{~h}$ of biopsy, the pneumothorax was more severe and more than $50 \%$ lung tissue was compressed (white arrows).

regression analysis, and regression coefficients and odds ratios $(\mathrm{OR})$ were calculated for independent risk factors. A pneumothorax risk prediction model was created using the regression coefficients to determine the predictive probability (PP) of pneumothorax.

$\mathrm{PP}=\frac{\exp (a+b 1 \times x 1+b 2 \times x 2+\ldots b n \times x n)}{1+\exp (a+b 1 \times x 1+b 2 \times x 2+\ldots b n \times x n)}$

A $\mathrm{P}$ value $<0.05$ was considered statistically significant. Statistical analysis was performed using statistical software (SPSS version 13.0, SPSS, Chicago, IL, USA).

\section{Results}

\section{General clinical data}

A total of 864 patients were included in this study and their general characteristics are presented in Table 1.

\section{Univariate analysis}

Of the 864 patients, 271 (31.4\%) showed varying degrees of pneumothorax. Univariate analysis showed that age, emphysema, small lesion size, no contact between the lesion and the pleura, prone or lateral body position, and multiple pleural punctures were significant risk factors of pneumothorax after biopsy (Table 1, Figure 2). In the group with lesions that had no contact between the lesion and the pleura ( $n=607)$, the ROC curve showed that the depth of the lesion had no statistically significant effect on the incidence of pneumothorax (AUC $=0.481, \mathrm{P}=0.448$ ) (Figure 3).

\section{Multivariate analysis}

The multivariate logistic regression analysis using six risk factors (age, emphysema, short-axis size of the lesion, contact between the lesion and the pleura, body position, 
Table 1 General clinical data and univariate analysis of pneumothorax risk factors

\begin{tabular}{|c|c|c|c|c|c|}
\hline Risk factor & Total & $\begin{array}{l}\text { Pneumothorax group } \\
\qquad(\mathrm{n}=271)\end{array}$ & $\begin{array}{l}\text { Non-pneumothorax group } \\
\qquad(\mathrm{n}=593)\end{array}$ & Statistic value & $P$ value \\
\hline Age (years) & $27-83(59.4 \pm 10.7)$ & $60.9 \pm 10.2$ & $58.8 \pm 10.7$ & $2.646^{\star}$ & 0.008 \\
\hline Emphysema & 271 (31.4\%) & $98(50.5 \%)$ & $96(49.5 \%)$ & $12.734^{\#}$ & 0.000 \\
\hline Depth $^{\wedge}(n=607), \mathrm{cm}$ & $0.1-10.3(2.4 \pm 1.5)$ & $2.3 \pm 1.4$ & $2.4 \pm 1.5$ & $0.758^{\star}$ & 0.449 \\
\hline \multicolumn{6}{|l|}{ Position } \\
\hline Supine & 364 (42.1\%) & $60(25.5 \%)$ & $175(74.5 \%)$ & & \\
\hline Single & $171(19.8 \%)$ & $30(17.5 \%)$ & 141 (82.5\%) & $18.920^{\#}$ & 0.000 \\
\hline Multiple & $693(80.2 \%)$ & 241 (34.8\%) & 452 (65.2\%) & & \\
\hline
\end{tabular}

Data are presented in range, mean $\pm \mathrm{SD}$ or $\mathrm{n}(\%){ }^{*}, \mathrm{t}$ value of $\mathrm{t}$-test; ${ }^{\#}, \chi^{2}$ value of $\chi^{2}$ test; $\wedge$, non-adjacent pleura group cases $($ depth $\neq 0)$.
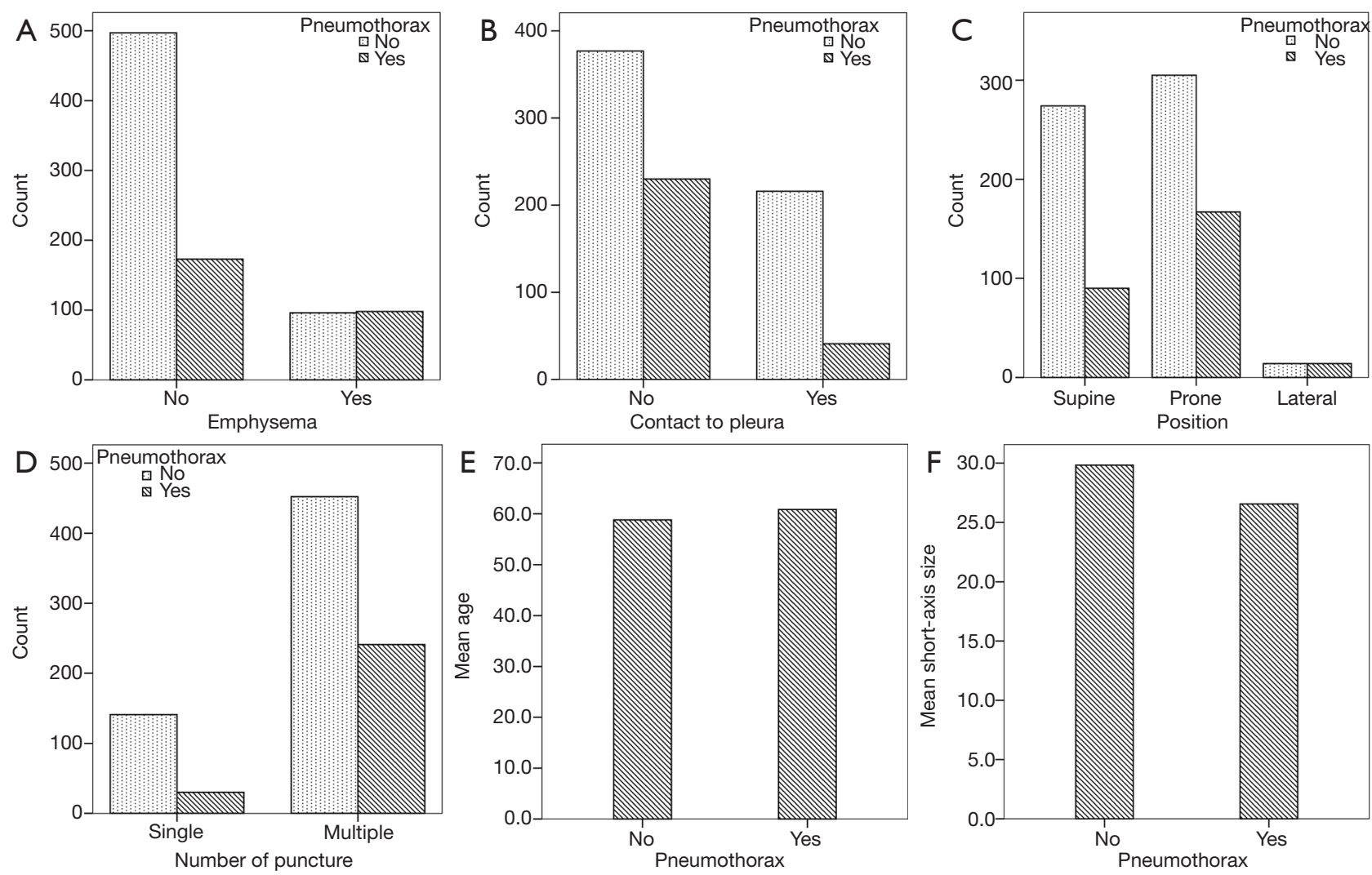

Figure 2 Bar graphs of incidence of pneumothorax with six risk factors: (A) emphysema, (B) contact between the lesion and the pleura, (C) body position, (D) number of pleural punctures, (E) age, and (F) short-axis size of the lesion. 
Table 2 Multivariate logistic regression analysis of pneumothorax risk factors

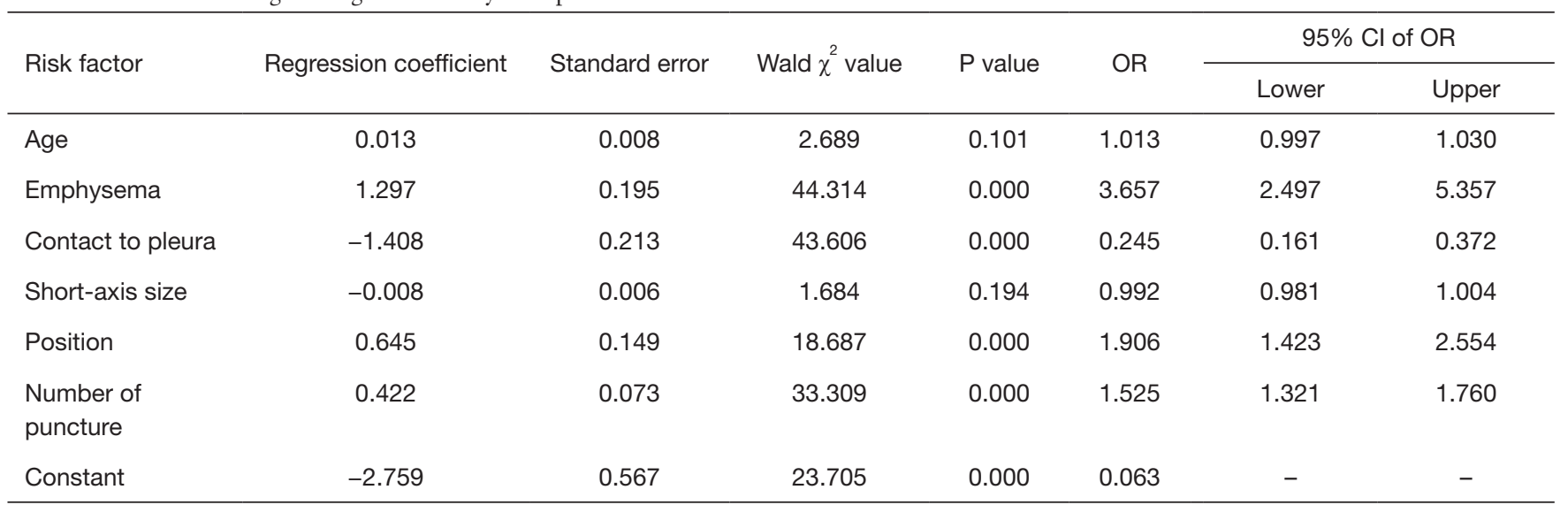

OR, odds ratios.

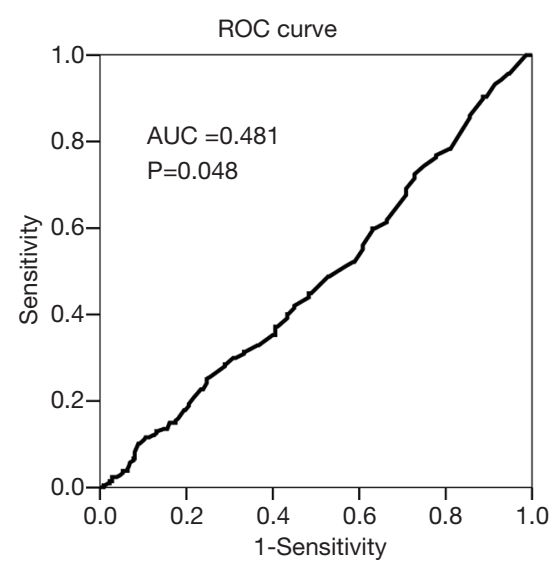

Figure 3 ROC curve of the relationship between depth of the lesion and incidence of pneumothorax $(n=607)$. ROC, receiver operating characteristic.

and number of pleural punctures) showed that emphysema, contact between the lesion the pleura, body position, and number of pleural punctures were each independent risk factors. Although age and short-axis size of the lesion were predictive of pneumothorax in the univariate analysis, they were not found to be independent risk factors of pneumothorax in the multivariate analysis (Table 2).

\section{Risk prediction model}

The risk prediction model of pneumothorax used the following regression analysis formula:

$\mathrm{PP}=\frac{\exp (0.013 \times A+1.297 \times B-1.408 \times C-0.008 \times D+0.645 \times E+0.422 \times F-2.759)}{1+\exp (0.013 \times A+1.297 \times B-1.408 \times C-0.008 \times D+0.645 \times E+0.422 \times F-2.759)}$ $1+\exp (0.013 \times A+1.297 \times B-1.408 \times C-0.008 \times D+0.645 \times E+0.422 \times F-2.759)$
Note: A, age; B, emphysema; C, contact between the lesion and the pleura; D, short-axis size of the lesion; E, body position; $\mathrm{F}$, number of pleural punctures.

The PP of pneumothorax and the incidence of pneumothorax were analyzed using the ROC curve (Figure 4) $(\mathrm{AUC}=0.735, \mathrm{P}=0.000)$. The sensitivity, specificity, and accuracy of the predictive model for pneumothorax were $56.8 \%, 79.6 \%$, and $72.5 \%$, respectively.

\section{Discussion}

Pneumothorax is the most common complication of transthoracic percutaneous needle biopsy, with a frequency of $24.0 \%$ to $59.6 \%$, whereas the incidence of pneumothorax requiring chest tube drainage ranges from $2.2 \%$ to $14.2 \%$ $(15,26,27,29,31,32)$. A large pneumothorax leads to lung collapse, and the risk to patients increases significantly when this occurs in an outpatient setting. The ability to predict the probability of a pneumothorax would be quite valuable when performing a CT-guided needle biopsy.

In the present study, the rate of pneumothorax was $31.4 \%$, which is comparable to previous studies. Our results showed that emphysema, no contact between the lesion and the pleura, prone or lateral body position, and multiple punctures were independent risk factors of pneumothorax. Most pervious literature suggests that age is an independent risk factor of pneumothorax; however, this may be due to emphysema being more common in older people. Thus, the increased risk of pneumothorax might be due to the presence of emphysema rather than age itself. Emphysema was found to be an independent risk factor of pneumothorax 


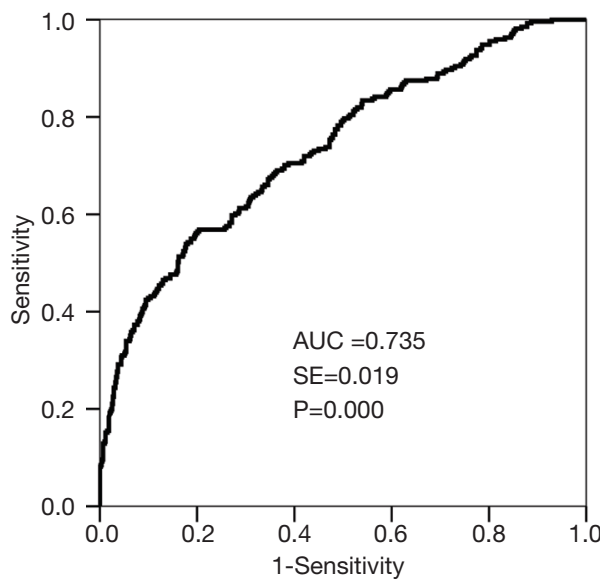

Figure 4 ROC curve of the relationship between predictive probability (PP) and the incidence of pneumothorax. ROC, receiver operating characteristic.

in this study ( $\mathrm{OR}=3.657)$, which is comparable to previous studies $(15,16,33)$. The increased risk of pneumothorax from emphysema is primarily due to decreased lung elasticity and poor retraction of the lungs, which can easily cause a bulla followed by a pneumothorax. Lesions in contact with the pleura essentially have a depth of $0 \mathrm{~cm}$, and previous studies found that the incidence of pneumothorax was higher when lesions were deeper with 1.0 or $2.0 \mathrm{~cm}$ used as cutoff points (15,16,30,33-35); however, all of those studies included a depth of $0 \mathrm{~cm}$ in the analysis. In this study, we analyzed 607 lesions that had no contact with the pleura (depth $\neq 0 \mathrm{~cm}$ ) and found that depth of the lesion was not a significant risk factor of pneumothorax. Hence, lesion depth itself is not a risk factor of pneumothorax if the lesion does not contact the pleura, although depth is a risk factor when there is contact between the lesion and the pleura. Kakizawa et al. (25) found that the multiple punctures also increased the incidence of pneumothorax, which is consistent with the results of our study (30/171 vs. $\left.241 / 693, \chi^{2}=18.920, \mathrm{P}=0.000\right)$; this might be due to greater damage to the pleura and alveoli.

Our study also found that being in the prone or lateral position was an independent risk factor of pneumothorax. Most previous studies have not described the influence of body position nor assumed that it had any relationship with pneumothorax, except for Nakamura (26) who thought that being in the supine position was an independent risk factor; Nakamura's suggestion is in contrast with the results of our study. It is difficult, however, to explain the influence of body position on the risk of pneumothorax.
Although lesion size was associated with pneumothorax in the univariate analysis, it was not shown to be an independent risk factor in the multivariate analysis. Only Hiraki (15) thought that the size of the lesion did not correlate with the risk of pneumothorax, which is consistent with the findings of our study. Other researchers have thought that the incidence of pneumothorax was correlated with small lesion size $(15,16,25,30,36)$. Because the accuracy of a biopsy is worse with smaller lesions, a higher number of pleural punctures may be required, which then increases the risk of pneumothorax.

Our study showed that the need for multiple biopsies, but not the size of the lesion, was a significant risk factor of pneumothorax. This result seems to contradict itself. However, most prior studies did find that performing multiple biopsies was a risk factor of pneumothorax, which is consistent with our findings (37-40).

Six risk factors, including age, emphysema, short-axis size of the lesion, contact between the lesion and the pleura, body position, and number of pleural punctures, were used for the pneumothorax risk prediction model. This model integrates multiple risk factors to determine the probability of pneumothorax. Although no prediction method can ever reach $100 \%$ accuracy, this model predicts pneumothorax with a high degree of accuracy (AUC $=0.735$ ). Furthermore, the prediction model proposed in our study has not been previously reported.

Our study does have several limitations. First, there is a risk of selection bias, as the majority of cases in this study, 768/864 (88.9\%), were performed in an out-patient setting; this may have skewed patient selection toward low-risk patients. Second, some risk factors, such as the puncture angle, were not included in this study. Finally, we cannot explain why body position significantly affected the incidence of pneumothorax.

In conclusion, emphysema, no contact between the lesion and the pleura, prone or lateral body position, and the need for multiple biopsies were independent risk factors of pneumothorax in this study. Patient age, depth of the lesion, and size of the lesion were not independent predictive factors of pneumothorax. Thus, the risk of pneumothorax can be predicted with a high degree of accuracy using the model designed in this study.

\section{Acknowledgements}

Funding: This work was supported by Beijing Hope Run Special Fund of China Cancer Research Foundation (CCRF) 
(No. LC2016A04)

\section{Footnote}

Conflicts of Interest: The authors have no conflicts of interest to declare.

Ethical Statement: This prospective study was approved by the Ethics Committee of our hospital (No. NCC2016YZ-15), and all patients signed an informed consent before the procedure.

\section{References}

1. Haaga JR, Alfidi RJ. Precise biopsy localization by computer tomography. Radiology 1976;118:603-7.

2. Li H, Boiselle PM, Shepard JO, et al. Diagnostic accuracy and safety of CT-guided percutaneous needle aspiration biopsy of the lung: comparison of small and large pulmonary nodules. AJR Am J Roentgenol 1996;167:105-9.

3. Connolly BL, Chait PG, Duncan DS, et al. CT-guided percutaneous needle biopsy of small lung nodules in children. Pediatr Radiol 1999;29:342-6.

4. Tsukada H, Satou T, Iwashima A, et al. Diagnostic accuracy of CT-guided automated needle biopsy of lung nodules. AJR Am J Roentgenol 2000;175:239-43.

5. Yeow KM, Tsay PK, Cheung YC, et al. Factors affecting diagnostic accuracy of CT-guided coaxial cutting needle lung biopsy: retrospective analysis of 631 procedures. J Vasc Interv Radiol 2003;14:581-8.

6. Shi JM, Cai Z, Huang H, et al. Role of CT-guided percutaneous lung biopsy in diagnosis of pulmonary fungal infection in patients with hematologic diseases. Int J Hematol 2009;89:624-7.

7. Tian P, Wang Y, Li L, et al. CT-guided transthoracic core needle biopsy for small pulmonary lesions: diagnostic performance and adequacy for molecular testing. J Thorac Dis 2017;9:333-43.

8. Wang YX, Gong JS, Suzuki K, et al. Evidence based imaging strategies for solitary pulmonary nodule. J Thorac Dis 2014;6:872-87.

9. Bahtouee M, Saberifard J, Nabipour I, et al. Combined computed tomography (CT)/scintigraphy strategy may help in diagnostic dilemmas in interstitial lung disease (ILD). Quant Imaging Med Surg 2016;6:460-1.

10. Wangkaew $S$, Euathrongchit J, Wattanawittawas $P$, et al. Correlation of delta high-resolution computed tomography (HRCT) score with delta clinical variables in early systemic sclerosis (SSc) patients. Quant Imaging Med Surg 2016;6:381-90.

11. Çetinçakmak MG, Göya C, Hamidi C, et al. Quantitative volumetric assessment of pulmonary involvement in patients with systemic sclerosis. Quant Imaging Med Surg 2016;6:50-6.

12. Anderson JM, Murchison J, Patel D. CT-guided lung biopsy: factors influencing diagnostic yield and complication rate. Clin Radiol 2003;58:791-7.

13. Charig MJ, Phillips AJ. CT-guided cutting needle biopsy of lung lesions--safety and efficacy of an out-patient service. Clin Radiol 2000;55:964-9.

14. Arslan S, Yilmaz A, Bayramgurler B, et al. CT- guided transthoracic fine needle aspiration of pulmonary lesions: accuracy and complications in 294 patients. Med Sci Monit 2002;8:CR493-7.

15. Hiraki T, Mimura $H$, Gobara $H$, et al. Incidence of and risk factors for pneumothorax and chest tube placement after CT fluoroscopy-guided percutaneous lung biopsy: retrospective analysis of the procedures conducted over a 9-year period. AJR Am J Roentgenol 2010;194:809-14.

16. Boskovic T, Stanic J, Pena-Karan S, et al. Pneumothorax after transthoracic needle biopsy of lung lesions under CT guidance. J Thorac Dis 2014;6:S99-107.

17. Ishii H, Hiraki T, Gobara H, et al. Risk factors for systemic air embolism as a complication of percutaneous CT-guided lung biopsy: multicenter case-control study. Cardiovasc Intervent Radiol 2014;37:1312-20.

18. Bhatia S. Systemic air embolism following CT-guided lung biopsy. J Vasc Interv Radiol 2009;20:709-11.

19. Capasso R, Nizzoli R, Tiseo M, et al. Extra-pleuric coaxial system for CT-guided percutaneous fine-needle aspiration biopsy (FNAB) of small $(</=20 \mathrm{~mm})$ lung nodules: a novel technique using multiplanar reconstruction (MPR) images. Med Oncol 2017;34:17.

20. Chen $L, Z$ Zhang $Z$. Bedside ultrasonography for diagnosis of pneumothorax. Quant Imaging Med Surg 2015;5:618-23.

21. Lee HY, Lee IJ. Assessment of Independent Risk Factors of Developing Pneumothorax During Percutaneous Core Needle Lung Biopsy: Focus on Lesion Depth. Iran J Radiol 2016;13:e30929.

22. Digumarthy SR, Kovacina B, Otrakji A, et al. Percutaneous CT guided lung biopsy in patients with pulmonary hypertension: Assessment of complications. Eur J Radiol 2016;85:466-71.

23. Wang Y, Jiang F, Tan X, et al. CT-guided percutaneous transthoracic needle biopsy for paramediastinal and nonparamediastinal lung lesions: Diagnostic yield and 
complications in 1484 patients. Medicine (Baltimore) 2016;95:e4460.

24. Ashraf H, Krag-Andersen S, Naqibullah M, et al. Computer tomography guided lung biopsy using interactive breath-hold control: a randomized study. Ann Transl Med 2017;5:253.

25. Kakizawa H, Toyota N, Hieda M, et al. Risk factors for severity of pneumothorax after CT-guided percutaneous lung biopsy using the single-needle method. Hiroshima J Med Sci 2010;59:43-50.

26. Nakamura M, Yoshizako T, Koyama S, et al. Risk factors influencing chest tube placement among patients with pneumothorax because of CT-guided needle biopsy of the lung. J Med Imaging Radiat Oncol 2011;55:474-8.

27. Yamagami T, Nakamura T, Iida S, et al. Management of pneumothorax after percutaneous CT-guided lung biopsy. Chest 2002;121:1159-64.

28. Traill ZC, Gleeson FV. Delayed pneumothorax after CTguided percutaneous fine needle aspiration lung biopsy. Thorax 1997;52:581-2; discussion 575-6.

29. Saji H, Nakamura H, Tsuchida T, et al. The incidence and the risk of pneumothorax and chest tube placement after percutaneous CT-guided lung biopsy: the angle of the needle trajectory is a novel predictor. Chest 2002;121:1521-6.

30. Asai N, Kawamura Y, Yamazaki I, et al. Is emphysema a risk factor for pneumothorax in CT-guided lung biopsy? Springerplus 2013;2:196.

31. Yamagami T, Kato T, Iida S, et al. Efficacy of manual aspiration immediately after complicated pneumothorax in CT-guided lung biopsy. J Vasc Interv Radiol 2005;16:477-83.

32. Chakrabarti B, Earis JE, Pandey R, et al. Risk assessment of pneumothorax and pulmonary haemorrhage complicating percutaneous co-axial cutting needle lung biopsy. Respir Med 2009;103:449-55.

33. Laurent F, Michel P, Latrabe V, et al. Pneumothoraces and chest tube placement after CT-guided transthoracic lung biopsy using a coaxial technique: incidence and risk factors. AJR Am J Roentgenol 1999;172:1049-53.

34. Yeow KM, See LC, Lui KW, et al. Risk factors for pneumothorax and bleeding after CT-guided percutaneous coaxial cutting needle biopsy of lung lesions. J Vasc Interv Radiol 2001;12:1305-12.

35. Min L, Xu X, Song Y, et al. Breath-hold after forced expiration before removal of the biopsy needle decreased the rate of pneumothorax in CT-guided transthoracic lung biopsy. Eur J Radiol 2013;82:187-90.

36. Nakatani M, Tanigawa N, Kariya S, et al. Analysis of factors influencing accuracy and complications in CTguided lung biopsy. Minim Invasive Ther Allied Technol 2012;21:415-22.

37. Taleb S, Jalaeian H, Frank N, et al. Is a Routine Chest X-ray Necessary in Every Patient After Percutaneous CTGuided Lung Biopsy? A Retrospective Review of 278 Cases. Cardiovasc Intervent Radiol 2017;40:1415-20.

38. Heerink WJ, de Bock GH, de Jonge GJ, et al. Complication rates of CT-guided transthoracic lung biopsy: meta-analysis. Eur Radiol 2017;27:138-48.

39. Nour-Eldin NE, Alsubhi M, Emam A, et al. Pneumothorax Complicating Coaxial and Non-coaxial CT-Guided Lung Biopsy: Comparative Analysis of Determining Risk Factors and Management of Pneumothorax in a Retrospective Review of 650 Patients. Cardiovasc Intervent Radiol 2016;39:261-70.

40. Moreland A, Novogrodsky E, Brody L, et al. Pneumothorax with prolonged chest tube requirement after CT-guided percutaneous lung biopsy: incidence and risk factors. Eur Radiol 2016;26:3483-91.
Cite this article as: Zhao Y, Wang X, Wang Y, Zhu Z. Logistic regression analysis and a risk prediction model of pneumothorax after CT-guided needle biopsy. J Thorac Dis 2017;9(11):47504757. doi: 10.21037/jtd.2017.09.47 